\title{
MEDICINE
}

\section{THROMBOTIC THROMBOCYTOPENIC PURPURA (TTP) AND MODERN APPROACH TO ITS INVESTIGATION AND TREATMENT}

\author{
K. Ukleba, L. Gvetadze, \\ Department of Clinical Medicine, Medical faculty, Akaki Tsereteli state University Kutaisi, Georgia
}

DOI: https://doi.org/10.31435/rsglobal_sr/31032019/6381

\section{ARTICLE INFO}

Received 16 January 2019

Accepted 16 March 2019

Published 31 March 2019

\section{KEYWORDS}

Thrombocytopenic Purpura, Microangiopatic hemolytic anemia,

ADAMTS13. \begin{abstract}
Thrombotic thrombocytopenic purpura (TTP) is a rare and life-threatening thrombotic microangiopathy characterized by microangiopathic hemolytic anemia, severe thrombocytopenia, and organ ischemia linked to disseminated microvascular platelet rich-thrombi. TTP is specifically related to a severe deficiency in ADAMTS13 (a disintegrin and metalloprotease with thrombospondin type 1 repeats, member 13), the specific von Willebrand factor-cleaving protease. ADAMTS13 deficiency is most frequently acquired via ADAMTS13 autoantibodies, but rarely, it is inherited via mutations of the ADAMTS13 gane. The first acute episode of TTP usually occurs during adulthood, with a predominant anti - ADAMTS13 autoimmune etiology. In rare cases, however, TTP begins as soon as childhood, with frequent inherited forms. TTP is 2 - fold more frequent in women, and its outcome is characterized by a relapsing tendency.
\end{abstract}

Citation: K. Ukleba, L. Gvetadze. (2019) Thrombotic Thrombocytopenic Purpura (TTP) and Modern Approach to its Investigation and Treatment. Science Review. 3(20). doi: 10.31435/rsglobal_sr/31032019/6381

Copyright: (C) 2019 K. Ukleba, L. Gvetadze. This is an open-access article distributed under the terms of the Creative Commons Attribution License (CC BY). The use, distribution or reproduction in other forums is permitted, provided the original author(s) or licensor are credited and that the original publication in this journal is cited, in accordance with accepted academic practice. No use, distribution or reproduction is permitted which does not comply with these terms.

Introduction. TTP is thought to be a rare disease. Over one series, the frequency was approximately 1 in 50000 hospital admissions. Over a 25 year period in the Sacramento, California region (population at risk, 1.2 million) at least 176 documented cases of TTP were reported. Analysis of a French national registry found that the rate of TTP in France was 13 cases per million population.

Untreated TTP has a mortality rate of as high as $90 \%$. With plasma exchange the mortality rate is reduced to $10-20 \%$. Patients with TTP have unusually large multimers of von willebrand factor (WF) in their plasma and the lack a plasma protease that is responsible for the breakdown of these ultralarge WF multimers. In the congenital form of TTP, mutations in the gene encoding this protease have been described. This protease has been isolated and cloned and is designated ADAMTS13. To make an accurate diagnosis, the clinician must recognice the similarity between thrombotic thrombocytopenic purpura (TTP) and hemolytic - uremic syndrome (Hus). The differential diagnosis also includes immune thrombocytopenic purpura (ITP) and disseminated intrawascular coagulation (Dic).

In addition to the microangiopathic hemolytic anemia and consumption thrombocytopenia, classical parameters for hemolysis show a high reticulocyte count (>120X109/L), an undetectable serum haptoglobin concentration, and an elevated lactate dehydrogenase level, a marker for tissue damage. The fresence of schistocytes on the blood smear (helmet cells; small, irregular triangular, or crescent -shaped cells; pointed projections; and lack of central pallor) with a confident threshold value of $1 \%$ is the morphologic hallmark of the disease. Except in some associated autoimmune contexts (SLE), the erythrocyte Coombs' test is negative, Standard coagulation parameters are usually normal. 
Renal testing may show proteinuria, hematuria, and sometimes increased plasma urea and creatinine levels. An increased cardiac troponin level $(>0.1 \mu$ is) is present in up to $60 \%$ of cases, the majority of whom have no clinical cardiac involvement. Electrocardiogram changes, mainly repolarization disorders, are present in $10 \%$ of cases. Point-based TTP prediction scores have been validated to predict an acquired ADAMTS13 deficiency. These scores include platelet count, serum creatinine level, and either detectable antinuclear antibodies or d-dimer, reticulocytes, and indirect bilirubin.

As these standard investigations are not specific for TTP and may be present in the miscellaneous differential diagnosis for TTP, they should be complemented by analysis of ADAMTS13, the unique marker sensitive and specific for TTP.

Clinical spectrum of TTP

Today, the historical clinical pentad of fever, thrombocytopenia, microangiopathic hemolytic anemia, neurological symptoms, and renal insufficiency that used to define TTP appears obsolete, as several cohort studies have clearly demonstrated that these 5 symptoms were present in less than $10 \%$ of patients with an acute TTP. The almost constant signs of TTP remain severe thrombocytopenia (typically $<30 \times 109 / \mathrm{L}$ ) and microangiopathic hemolytic anemia characterized by schistocytes on the blood smear, often associated with corresponding symptoms (ie, skin and mucosal hemorrhage, weakness, and dispnoe). Symptoms related to o ischemia/infarction mostly concern the brain (-60\% of patients have neurologic symptoms at presentation, with a broad range from headache and confusion to stroke, coma, and seizures). Heart ischemia is also frequent (-25\% of patients, ranging from isolated electrocardiographic abnormalities to myocardial infarction). as well as mesenteric ischemia (-35\%) causing abdominal pain and seizures. Heart ischemia is also freqienr $(-25 \%)$ of an isolated proteinuria/hematuria; acute renal failure is unusual in TTP, with typically a serum creatinine level below $2 \mathrm{mg} / \mathrm{dL}$ at presentation.

Rapid recognition of TTP is crucial to initiate appropriate treatment. The first-line therapy for acute TTP is sased on daily therapeutic plasma exchange supplying deficient ADAMTS13, with or without steroids. Additional immune modulators targeting ADAMTS13 autoantibodies are mainly based on steroids and humanized anti-CD20 monoclinal antibody rituximab. In refractory or unresponsive TTP, more intensive tharapies including twice-daily plasma exchange; pulses of cyclophosphamide, vincristine, or cyclosporine A; salvage splenectomy are considered. New drugs including $\mathrm{N}$-acetylcysteine, bortezomib, recombinant ADAMTS13, and caplacizumab show promise in the management of TTP. Also, long-term follow-up of patients with TTP is crucial to identisy the occurrence of other autoimmune diseases, to control relapses, and to evaluate psychophysical sequelae. The overall response rate to plasma exchange is $75-90 \%$. The early mortality rate is $10-20 \%$.

\section{REFERENCES}

1. Kremer Hovinga, JA; Coppo, P; Lämmle, B; Moake, JL; Miyata, T; Vanhoorelbeke, K (6 April 2017). "Thrombotic thrombocytopenic purpura". Nature Reviews. Disease Primers. 3: 17020. doi:10.1038/nrdp.2017.20. PMID 28382967.

2. "Thrombotic thrombocytopenic purpura, acquired". Genetic and Rare Diseases Information Center (GARD) - an NCATS Program. Retrieved 10 October 2018.

3. Joly, BS; Coppo, P; Veyradier, A (25 May 2017). "Thrombotic thrombocytopenic purpura". Blood. 129 (21): 2836-2846. doi:10.1182/blood-2016-10-709857. PMID 28416507.

4. George JN (November 2010). "How I treat patients with thrombotic thrombocytopenic purpura: 2010". Blood. 116 (20): 4060-9. doi:10.1182/blood-2010-07-271445. PMID 20686117.

5. Moake JL (2004). "von Willebrand factor, ADAMTS-13, and thrombotic thrombocytopenic purpura". Semin. Hematol. 41 (1): 4-14. doi:10.1053/j.seminhematol.2003.10.003. PMID 14727254.

6. Wood, Marie E.; Philips, George K. (2003). Hematology/oncology Secrets. Elsevier Health Sciences. p. 68. ISBN 978-1560535164.

7. Shatzel, JJ; Taylor, JA (March 2017). "Syndromes of Thrombotic Microangiopathy". The Medical Clinics of North America(Review). 101 (2): 395-415. doi:10.1016/j.mcna.2016.09.010. PMID 28189178.

8. Allford S, Machin S (2005). "Thrombotic thrombocytopenic purpura". NetDoctor.co.uk.

9. Moake JL (2002). "Thrombotic microangiopathies". N. Engl. J. Med. 347 (8): 589600. doi:10.1056/NEJMra020528. PMID 12192020.

10. Moake JL (1998). "Moschcowitz, multimers, and metalloprotease". N. Engl. J. Med. 339 (22): 162931. doi:10.1056/NEJM199811263392210. PMID 9828253.

11. Furlan M, Robles R, Galbusera M, et al. (1998). "von Willebrand factor-cleaving protease in thrombotic thrombocytopenic purpura and the hemolytic-uremic syndrome". N. Engl. J. Med. 339 (22): 157884. doi:10.1056/NEJM199811263392202. PMID 9828245.

12. Tsai HM, Lian EC (1998). «Antibodies to von Willebrand factor-cleaving protease in acute thrombotic thrombocytopenic purpura»». N. Engl. J. Med. 339 (22): 1585-94. doi:10.1056/NEJM199811263392203. PMC 3159001. PMID 9828246. 